\title{
Identification of genetic variants of lecithin cholesterol acyltransferase in individuals with high HDL-C levels
}

\author{
MOHSEN NASERI ${ }^{1}$, MEHDI HEDAYATI ${ }^{1}$, MARYAM SADAT DANESHPOUR $^{1}$, \\ FATEMEH BANDARIAN $^{1}$ and FEREIDOUN AZIZI ${ }^{2}$
}

\begin{abstract}
${ }^{1}$ Cellular and Molecular Endocrine Research Center; ${ }^{2}$ Endocrine Research Center, Research Institute for Endocrine Sciences, Shahid Beheshti University of Medical Sciences, Tehran, Iran
\end{abstract}

Received September 18, 2013; Accepted March 5, 2014

DOI: $10.3892 / \mathrm{mmr} .2014 .2177$

\begin{abstract}
Among the most common lipid abnormalities, a low level of high-density lipoprotein-cholesterol (HDL-C) is one of the first risk factors identified for coronary heart disease. Lecithin cholesterol acyltransferase (LCAT) has a pivotal role in the formation and maturation of HDL-C and in reverse cholesterol transport. To identify genetic loci associated with low HDL-C in a population-based cohort in Tehran, the promoter, coding regions and exon/intron boundaries of LCAT were amplified and sequenced in consecutive individuals $(n=150)$ who had extremely low or high HDL-C levels but no other major lipid abnormalities. A total of 14 single-nucleotide polymorphisms (SNPs) were identified, of which 10 were found to be novel; the L393L, S232T and 16:67977696 C>A polymorphisms have been previously reported in the SNP Database (as rs5923, rs4986970 and rs11860115, respectively) and the non-synonymous R47M mutation has been reported in the Catalogue of Somatic Mutations in Cancer (COSM972635). Three of the SNPs identified in the present study (position 6,531 in exon 5, position 6,696 in exon 5 and position 5,151 in exon 1) led to an amino acid substitution. The most common variants were L393L (4886C/T) in exon 6 and Q177E, a novel mutation, in exon 5, and the prevalence of the heterozygous genotype of these two SNPs was significantly higher in the low HDL-C groups. Univariate conditional logistic regression odds ratios (ORs) were nominally significant for Q177E (OR, 5.64; $\mathrm{P}=0.02 ; 95 \%$ confidence interval, 1.2-26.2). However, this finding was attenuated following adjustment for confounders. Further studies using a larger sample size may enhance the determination of the role of these SNPs.
\end{abstract}

Correspondence to: Professor Fereidoun Azizi, Endocrine Research Center, Research Institute for Endocrine Sciences, Shahid Beheshti University of Medical Sciences, Parvaneh Avenue, Yaman Street, Chamran Highway, Tehran, PO Box 19195-4763, Iran E-mail: azizi@endocrine.ac.ir

Key words: lecithin cholesterol acyltransferase, high-density lipoproteins, single nucleotide polymorphism, mutation

\section{Introduction}

Cardiovascular diseases (CVDs) are the primary cause of mortality worldwide (1). The prevalence of cardiovascular risk factors, particularly low high-density lipoprotein-cholesterol (HDL-C) levels, was high (32\%) in participants of the Tehran Lipid and Glucose Study (TLGS) (2). Several previous studies have found an inverse correlation between plasma HDL-C and the risk of CVDs $(3,4)$. Since HDL-C levels are predominantly under genetic control, with heritability estimates of $>80 \%$ (5), the detection and characterization of genetic variants associated with HDL-C concentration may provide novel insights in the field of life expectancy. A large number of genes contributing to HDL-C concentration have been identified; however, the variants detected in these genes together explain only a small amount $(<10 \%)$ of the HDL-C variances (6). Therefore, more studies are required to identify genetic variants, and this may be achieved by sequence-based approaches on the identified genes (6).

While loss-of-function mutations in apolipoprotein A-I (APOA1), LCAT or ATP cassette transporter A1 (ABCA1) cause monogenic HDL-C deficiencies, single gene defects in hepatic lipase (LIPC) or cholesteryl ester transfer protein (CETP) are associated with high HDL-C levels in humans (7,8). LCAT, first described in 1962 (9), is a soluble enzyme that has a central role in the formation and maturation of HDL-C. The majority of the LCAT is synthesized by the liver and circulates in the blood, reversibly bound to lipoproteins. In the blood, LCAT preferentially binds to HDL and is then activated by apoA-I, the major protein component of HDL, and converts phosphatidylcholines and cholesterol of HDL into lysophosphatidylcholines and cholesteryl esters (10). The LCAT gene, located on chromosome 16q22.1 reverse strand, spans 4,382 bp (11) and contains six exons with $\sim 1.5 \mathrm{~kb}$ of coding sequence that encodes a 416-amino acid protein (12).

Mutations in the LCAT gene have been found to cause fish-eye disease, as well as familial LCAT deficiency (13). A meta-analysis genome-wide association study (GWAS) on $>100,000$ individuals (14) and a Mendelian randomization study identified a single-nucleotide polymorphism (SNP) in LCAT as the strongest indicator of isolated variation in levels of HDL-C (15). 
The present study was performed to evaluate the role of genetic variation in LCAT (a biological candidate gene involved in HDL metabolism) in relation to HDL-C levels in epidemiological samples from the TLGS.

\section{Materials and methods}

Study population. In this cross-sectional study, 150 individuals, aged between 15 and 75 years, with HDL-C levels $<5$ th percentile adjusted for age and gender (84 individuals) and those with levels of HDL-C $>95$ th percentile adjusted for age and gender (66 individuals), without any other lipid abnormalities, were selected among TLGS participants. The TLGS is a prospective population-based longitudinal cohort study with $>15,000$ participants designed to determine the risk factors for major noncommunicable disorders in Tehran (16).

To increase the likelihood of genetic causes, only individuals who had the same trait in all four TLGS phases consistently and had at least one family member with the same trait were included. Obese individuals [body mass index $(\mathrm{BMI}) \geq 30 \mathrm{~kg} / \mathrm{m}^{2}$ ] and those receiving drugs affecting HDL-C levels were excluded from the study. A standardized questionnaire was used to collect data on demographics and medication usage for lipid disorders and hypertension treatment.

The study was conducted at the Research Institute for Endocrine Sciences in Shahid Beheshti University of Medical Sciences (Tehran, Iran) following approval by the Institutional Ethics Committee. Each participant signed a written informed consent form.

Biochemical assessment. Details of collection, preparation and lipid determination methods, together with details regarding quality control have been previously published (17). Briefly, a venous blood sample was obtained from each subject, and fasting blood glucose (FBS), total cholesterol (TC), triglycerides (TG), low-density lipoprotein cholesterol (LDL-C) and HDL-C were measured using a Selectra 2 Auto-Analyzer (Vital Scientific, Spankeren, The Netherlands) the same day. TC and TG were measured using the cholesterol enzymatic colorimetric assay kit (Pars Azmoon Inc., Tehran, Iran). Serum HDL-C levels were measured following precipitation of apo B-containing lipoproteins with dextran-magnesium sulfate (18). LDL-C was calculated in serum samples with TG $<400 \mathrm{mg} / \mathrm{dl}$ using the Friedwald formula (19).

Gene resequencing. In order to analyze the genetic polymorphisms of LCAT, genomic DNA was isolated from the blood of the participants using the standard proteinase $\mathrm{K}$ salting-out method (20). Sequences of human LCAT coding regions (GenBank NM_000229.1) were acquired from the University of California, Santa Cruz (UCSC) Genome Browser (http://genome.ucsc.edu/) (21). Six DNA fragments were amplified, covering 857 bp upstream of exon 1, all six exons of LCAT and the exon-intron boundaries. Primers were designed using web-based Primer3 software (22) and National Center for Biotechnology Information (NCBI) primer blast programs (23). Primers were verified using electronic PCR on the UCSC Genome Browser to ensure one unique genomic hit, and were also inspected for known SNPs using the SNP
Database (dbSNP) (24) and Ensembl (25). Polymerase chain reaction (PCR) conditions and primer sequences are provided in Table I.

Each PCR amplification was performed with $100 \mathrm{ng}$ genomic DNA in a 30- $\mu$ l final volume reaction, containing $40 \mathrm{pmol}$ each primer, $0.2 \mathrm{mmol} / 1$ each deoxynucleotide triphosphate, $1.5 \mathrm{mmol} / 1 \mathrm{MgCl}_{2}, 10 \mathrm{mmol} / 1$ Tris ( $\mathrm{pH} \mathrm{8.4)}$ and 0.25 units Taq polymerase (Fermentase Co., Burlington, ON, Canada). Hybridization was conducted in a DNA Thermal Cycler (Corbett Life Sciences, Sydney, Australia), in which, following pre-denaturation for $5 \mathrm{~min}$ at $96^{\circ} \mathrm{C}$, there were 32 cycles of $96^{\circ} \mathrm{C}$ for $50 \mathrm{sec}$, an annealing step at the temperature given for the primer pair (Table I) for $35 \mathrm{sec}$ and an elongation step at $72^{\circ} \mathrm{C}$ for $50 \mathrm{sec}$, followed by a final extension at $72^{\circ} \mathrm{C}$ for $5 \mathrm{~min}$. The PCR products were run on $1.5 \%$ agarose gel and the gel was then treated with $1 \mathrm{mg} / \mathrm{dl}$ ethidium bromide solution for $10 \mathrm{~min}$. The DNA fragments were subsequently visualized using the gel documentation system OptiGo (Isogen Life Science, De Meern, Netherlands).

Purification and sequencing of the PCR product. The PCR product was purified using the Qiagen kit (Germantown, MD, USA) in order to eliminate excess nucleotides, dimers and nonspecific bands, and the PCR product was then processed through direct sequencing using an Applied Biosystems 3730/3730xl DNA Analyzer (Applied Biosystems, Foster City, CA, USA). Sequence alignment analysis was performed using the Basic Local Alignment Search Tool (BLAST) (26), ClastalW (http://www.ebi.ac.uk/clustalw/) and chromas programs.

Statistical analysis. The allele frequency and consistency with the Hardy-Weinberg equilibrium were calculated using the PowerMarker software version 3.25 (27). The SPSS Version 15 software package (SPSS, Inc., Chicago, IL, USA) and STATA version 9.1 (StataCorp LP, College Station, TX, USA) were used to analyze the data. Categorical variable distribution was analyzed using the $\chi^{2}$ test. Multivariate logistic regressions were used to control the effects of potential confounders, including age, BMI, smoking and gender. For all tests, $\mathrm{P}<0.05$ was considered to indicate a statistically significant difference.

\section{Results}

Characteristics of the study population. The mean ages of individuals in the low and high HDL-C groups were $41 \pm 13$ and $37 \pm 16$ years, respectively. Table II summarizes the demographic and biochemical parameters, as well as the clinical characteristics of the studied population. The BMI, FBS, TC, TG and LDL-C in the low HDL-C group were significantly different from those in the high HDL-C group.

Identification of SNPs in the LCAT gene. The region $(\sim 1,500 \mathrm{bp})$ of the LCAT gene encoding 440 amino acids was determined by sequencing and NCBI BLAST. A total of 14 SNPs were identified through the sequencing of 300 chromosomes (Fig. 1). The type and position of the SNPs, as well as their frequency of occurrence, are listed in Table III. Six of the nucleotide substitutions were found to be in exons, whilst six SNPs occurred in introns and two were located in the 
Table I. Polymerase chain reaction primers used for the analysis of the lecithin cholesterol acyltransferase gene.

\begin{tabular}{|c|c|c|c|}
\hline Primer name & Primer sequence (5' to $3^{\prime}$ ) & Annealing $\left({ }^{\circ} \mathrm{C}\right)$ & Product size (bp) \\
\hline Promoter & $\begin{array}{l}\text { F: TGTTGCCTCCTTGACTTGAG } \\
\text { R: GGGAAGAGCACATTGAGGAG }\end{array}$ & 53 & 957 \\
\hline Exon 1 & $\begin{array}{l}\text { F: CCTTTCCGGCAATCTCTGa } \\
\text { R: TCACAGTGTGGTGGGAGAAG }\end{array}$ & 56 & 481 \\
\hline Exons 2 and 3 & $\begin{array}{l}\text { F: CCAGACTGGGTGTTTGCTC } \\
\text { R: TGTGTGCAGGTACCCTGTG }\end{array}$ & 59 & 731 \\
\hline Exon 4 & $\begin{array}{l}\text { F: AGCAAGCTGGCAGGTTTGTGTCA }{ }^{a} \\
\text { R: AAGACAGGCTTCCCATAGGCAG }\end{array}$ & 63 & 362 \\
\hline Exon 5 & $\begin{array}{l}\text { F: AACAATGGCTACGTGCGGGACGA }{ }^{a} \\
\text { R: AGTGGTAGATAGCACCCCTAGAG }\end{array}$ & 62 & 485 \\
\hline Exon 6 & $\begin{array}{l}\text { F: TGAGCCTACACTCAGCAGGTTGTG } \\
\text { R: CCCATCTTGCCTCACTGCACACA }\end{array}$ & 69 & 781 \\
\hline
\end{tabular}

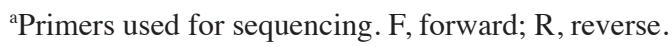

Table II. Characteristics of the study population.

\begin{tabular}{lccc}
\hline Variables & Subjects with high HDL-C & Subjects with low HDL-C & P-value \\
\hline Gender, M/F $(\% / \%)$ & $59.1 / 40.9$ & $75 / 25$ & NS \\
Age $($ years $)$ & $37 \pm 16$ & $41 \pm 13$ & NS \\
TC $(\mathrm{mg} / \mathrm{dl})$ & $194 \pm 42$ & $168 \pm 47$ & 0.001 \\
LDL-C $(\mathrm{mg} / \mathrm{dl})$ & $104 \pm 35$ & $90 \pm 28$ & 0.012 \\
TG $(\mathrm{mg} / \mathrm{dl})$ & $81 \pm 42$ & $251 \pm 179$ & $<0.001$ \\
BMI $\left(\mathrm{kg} / \mathrm{m}^{2}\right)$ & $23 \pm 3$ & $26 \pm 3$ & $<0.001$ \\
FBS $(\mathrm{mg} / \mathrm{dl})$ & $93 \pm 9$ & $108 \pm 34$ & 0.001 \\
SBP $(\mathrm{mmHg})$ & $113 \pm 19$ & $114 \pm 17$ & NS \\
DBP $(\mathrm{mmHg})$ & $73 \pm 11$ & $77 \pm 9$ & 0.016
\end{tabular}

Demographics and lipid levels among subjects with high and low HDL-C levels are shown. HDL-C, high-density lipoprotein cholesterol; TC, total cholesterol; LDL-C, low-density lipoprotein cholesterol; TG, triglycerides; BMI, body mass index; FBS, fasting blood glucose; SBP, systolic blood pressure; DBP, diastolic blood pressure; NS, not significant.

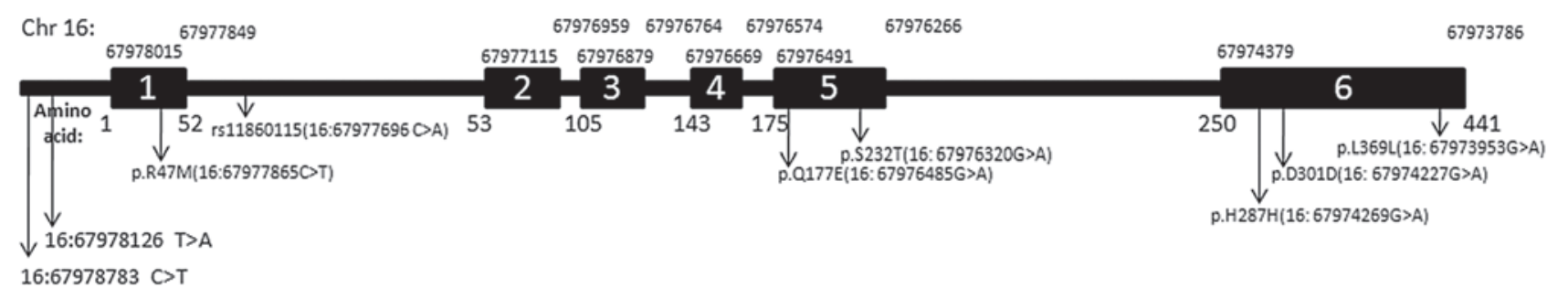

Figure 1. Structural diagram of the lecithin cholesterol acyltransferase gene showing the position of important single-nucleotide polymorphisms identified. Black boxes indicate exons 1-6. Nucleotide numbering reflects chromosome (NC_000016.9) and amino acid numbering is according to GenBank: AAB34898.1.

promoter. The nucleotide variations in the $5^{\prime}$ regulatory region occurred in one gene copy. These substitutions included a $\mathrm{C}$ instead of $\mathrm{T}$ at position $4,233(\mathrm{~g} .4233 \mathrm{~T}>\mathrm{A})$ and $\mathrm{G}$ instead of A at position $4,890(\mathrm{~g} .4890 \mathrm{C}>\mathrm{T})$ (positions given according to GenBank entry NG_009778.1).
Three of the SNPs in the protein coding regions were non-synonymous, whilst the others were synonymous. One SNP in exon 1 was observed in only one individual. No polymorphisms were found in exon 2. Three of the SNPs were in exon 6, two of which were synonymous and one of which was 


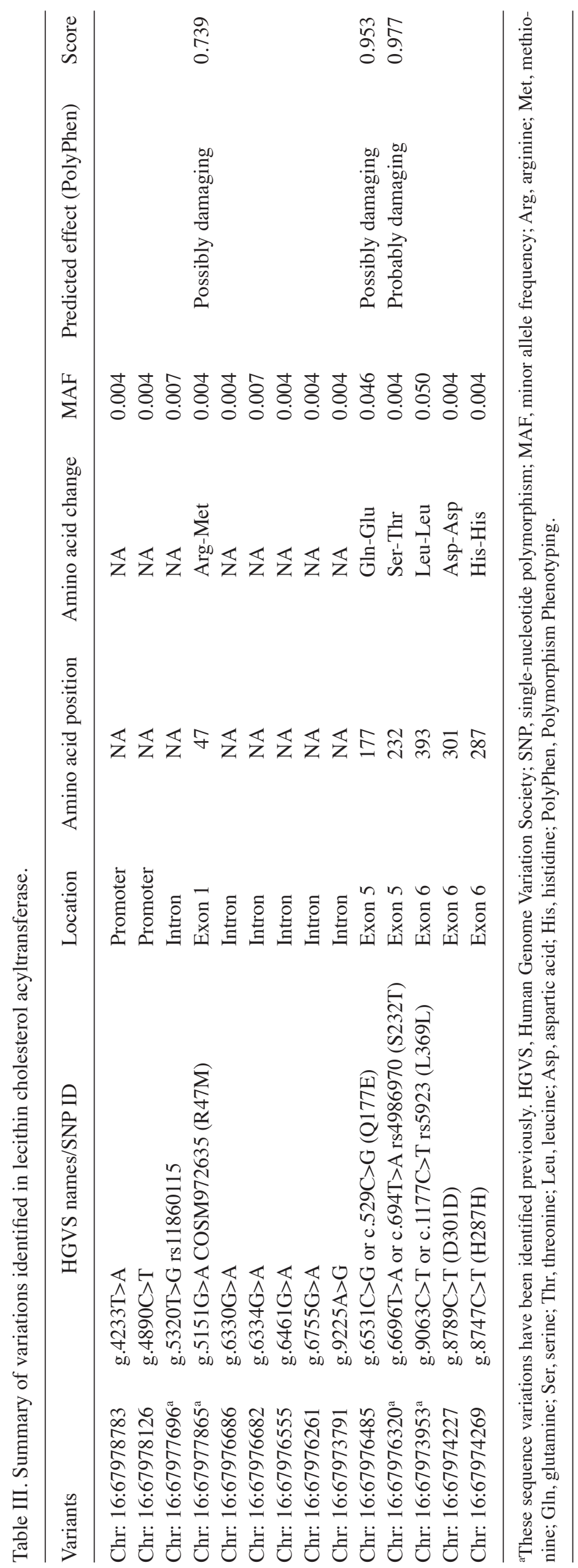


Table IV. Allele frequencies of lecithin cholesterol acyltransferase variants.

\begin{tabular}{|c|c|c|c|c|}
\hline \multirow[b]{2}{*}{ Number } & \multirow[b]{2}{*}{ Variant } & \multicolumn{2}{|c|}{ Allele frequency (\%) } & \multirow[b]{2}{*}{ P-value ${ }^{a}$} \\
\hline & & High HDL group & Low HDL group & \\
\hline 1 & Chr: 16:67978783 C>T & 0.9 & 0.0 & 0.41 \\
\hline 2 & Chr: 16:67978126 G>A & 0.0 & 0.6 & 0.52 \\
\hline 3 & Chr: 16:67977696 A>C & 0.0 & 1.2 & 0.37 \\
\hline 4 & Chr: 16:67977865 C> T & 0.0 & 0.6 & 0.52 \\
\hline 5 & Chr: 16:67976687 G> T & 1.5 & 0.0 & 0.44 \\
\hline 6 & Chr: 16:67976682 C > T & 0.0 & 1.2 & 0.37 \\
\hline 7 & Chr: 16:67976555 C> T & 0.9 & 0.0 & 0.41 \\
\hline 8 & Chr: $16: 67976261 \mathrm{C}>\mathrm{T}$ & 0.0 & 0.6 & 0.52 \\
\hline 9 & Chr: $16: 67973791 \mathrm{~T}>\mathrm{C}$ & 0.0 & 0.6 & 0.52 \\
\hline 10 & Chr: 16:67976485 G>C & 1.5 & 7.5 & 0.09 \\
\hline 11 & Chr: 16:67976320 A> T & 0.9 & 0.0 & 0.41 \\
\hline 12 & Chr: 16:67973953 G>A & 2.6 & 8.2 & 0.16 \\
\hline 13 & Chr: 16:67974227 G>A & 0.9 & 0.0 & 0.41 \\
\hline 14 & Chr: 16:67974269 G>C & 0.9 & 0.0 & 0.41 \\
\hline
\end{tabular}

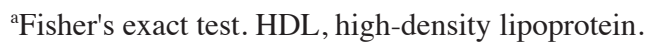

non-synonymous. Whilst all nucleotide substitutions in the 5 sequences were transition mutations, two of the substitutions within the coding sequences and two of the substitutions within the intron sequences were transversions.

A review of the dbSNP found that, to date (May 2013), 161 SNPs in the human LCAT gene have been submitted (24). Additionally, the Ensembl Genome Browser (25) has indexed 1,029 variants in the human LCAT gene with 1,136 downstream gene variants and 1,872 upstream gene variants (http://www. ensembl.org/Homo_sapiens/Gene/Variation_Gene/Table?g= ENSG00000213398; $\mathrm{r}=16: 67973653-67978034)$. In addition, $>80$ causative mutations in LCAT have been described (28) in the Human Gene Mutation Database (HGMD; http://www. hgmd.cf.ac.uk/ac/) (29).

Ten of the identified SNPs were found to be novel. The L393L, S232T and 16:67977696 C>A polymorphisms have been previously reported in the dbSNP Human Build 137 (as rs5923, rs4986970 and rs11860115, respectively), and the non-synonymous R47M mutation (COSM972635) was observed in Ensembl (release 18, 2013) and has been reported in the Catalogue of Somatic Mutations in Cancer (http://cancer. sanger.ac.uk/cosmic/mutation/overview?id=972635) (30).

Six of the 14 polymorphisms were found only in the high-HDL-C group, whilst six were only in the low-HDL-C group. Three of the 150 individuals carried two of the SNPs of LCAT. The most common variant was L393L in exon 6, found in 15 of 300 alleles, and the Q177E in exon 5, found in 14 of 300 alleles. Allele frequencies of low and high HDL-C groups are shown in Table IV. No significant differences were detected between the study groups. Evaluating genotype frequencies in low versus high HDL-C groups using Fisher's exact test demonstrated a significant association for the synonymous L393L $(\mathrm{P}=0.04)$ and the non-synonymous $\mathrm{Q} 177 \mathrm{E}(\mathrm{P}=0.02)$ SNPs. Univariate conditional logistic regression odds ratios
(ORs) were nominally significant for Q177E models only [OR, 5.64; $\mathrm{P}=0.02 ; 95 \%$ confidence interval (CI), 1.2-26.2]. Multivariate logistic models with adjustment for age, gender, smoking, FBS and BMI attenuated the effect (OR, 2.58; $\mathrm{P}=0.47$; $95 \% \mathrm{CI}, 0.16-39.5$ ).

Three of the identified SNPs (position 6,531 in exon 5, position 6,696 in exon 5 and position 5,151 in exon 1) gave rise to amino acid substitutions. Computational tools, for example Sorting Intolerant From Tolerant (31) and Polymorphism Phenotyping (PolyPhen) (32) are able to predict $90 \%$ of damaging SNPs. Table III shows analysis using PolyPhen, which revealed that two of the missense SNPs were possibly damaging, and one of them is probably damaging (available at http://genetics.bwh.harvard.edu/pph2/index.shtml).

\section{Discussion}

In the present study, enzymatic amplification and direct sequencing of the LCAT gene from individuals with high and low HDL-C were performed in order to investigate the association between LCAT and HDL-C levels in a population-based study in Tehran. Since LCAT is a biological candidate for HDL-C levels, the common and/or rare variants of LCAT are hypothesized to contribute to variation in HDL-C levels between individuals

To date, candidate genes, genome-wide linkage and GWASs have identified several genetic variations that may account for plasma HDL-C levels (8). GWASs independently identified numerous genetic loci for HDL-C (33-35). For example, eight GWASs analyzing HDL-C levels have confirmed the following genes that were recognized from functional studies: LCAT, CETP, LIPC, lipoprotein lipase, ABCA1, endothelial lipase and the gene cluster APOA1C3A4A5 (6). A meta-analysis GWAS, including $>100,000$ individuals, identified an SNP in 
the LCAT gene as the strongest marker of isolated variation in HDL-C levels (14). This indicates that variants in the LCAT gene associated with levels of HDL-C may be useful for a formal causality test of HDL-C levels for determining the risk of CVDs (15).

The genotyping platforms typically used in GWASs include mostly common variants [minor allele frequency (MAF) $>0.05$ ] that are unlikely to tag most of the rare variants in the genome or to identify the true causative variant due to the small effect sizes of common variants. One of the strategies used to identify these rare variants is the resequencing of extreme phenotypes, for which individuals at both extremes of the phenotype (e.g. highest and lowest five percentiles) are selected for further sequencing. These individuals may be part of a population-based cohort or a case-control study (36). Variants with major phenotypic effects are likely to be found only at one extreme, while alleles found in both the high and low HDL-C groups are most likely neutral with respect to HDL-C levels (37). Resequencing, unlike GWASs, requires a decision as to which gene to target. Resequencing is usually used to analyze the regions within a gene rather than the region between genes, and within exons rather than introns, and it is able to detect not only SNPs, but also structural variations such as copy-number variations (deletions and insertions) and copy-neutral variants (inversions and translocations) (36). Furthermore, resequencing requires smaller sample sizes, and previous studies have obtained positive results with initial sample sizes of $\leq 200$ (100 from each phenotypic extreme) (38). These positive findings may then be followed by studies on populations enriched for the variants, for example families of individuals who carry the risk alleles.

Cohen et al (37) resequenced three candidate genes (ABCA1, APOA1 and LCAT) in 256 individuals constituting the upper and lower $5 \%$ of the distribution of plasma HDL-C levels in a population-based study. The number of synonymous variants was similar in high and low HDL-C groups. Non-synonymous sequence variants were significantly more common (16 vs. $2 \%$ ) in individuals with low HDL-C compared with those with high HDL-C (six LCAT non-synonymous sequence variations were unique to the low-HDL-C group) (37). Therefore, it was concluded in the study that multiple rare, non-synonymous sequence variants of the genes that are involved in HDL metabolism may influence HDL-C levels. The results of the study supported the "rare variant-common disease' hypothesis (39).

In the present study, a similar approach was used as Cohen et al (37); however, the entire gene and flanking regions were sequenced so that the regulatory variants were also identified. A total of 150 individuals with HDL-C levels in the extremes were sequenced, and 14 variants were identified. Three of the variants were non-synonymous sequence variants, of which one was significantly more common in individuals with low HDL-C compared with those with high HDL-C. The other two were present only in the high HDL-C group. Three synonymous mutations were found in exon 6; only one of these mutations had an MAF of 5\%, and this mutation was more common in the low HDL-C group. The majority of identified variants $(71.4 \%)$ were absent from the dbSNP Human Build 137; however, some of them $(21.4 \%)$ were present in the 1,000 Genomes Project (http://www.1000genomes.org) and one SNP (COSM972635) was present in Ensembl (24). A synonymous $\mathrm{H} 287 \mathrm{H}$ mutation in the coding region of exon 6 of the LCAT gene was observed in an individual with HDL-C levels of $75 \mathrm{mg} / \mathrm{dl}$. CD952176 is the accession number of the coding sequence variant from the HGMD-PUBLIC dataset December 2012 (release 2012.4) that is present at 16:67974268. A previous study (40) demonstrated that point mutations (deletion or frameshift mutations) at this position result in LCAT deficiency.

Tietjen et al (41) resequenced 178 unrelated probands with HDL-C $<10$ th percentile and showed that mutations were most frequent in LCAT, followed by ABCA1 and APOA1; together, these explain $>40 \%$ of familial hypoalphalipoproteinemia. Furthermore, they observed a clear elevation in coronary artery disease risk among individuals with the mutations and a large reduction in HDL-C (41). Holleboom et al (28) found high mutation frequencies of LCAT in individuals with low levels of HDL-C in the Netherlands, and $50 \%$ of the LCAT mutations were a p.T147I mutation (28).

Due to time constraints and technical issues in the present study, the identified variants were not genotyped. When all variants are genotyped in the entire population, it may be possible to determine the extent to which LCAT variants influence HDL-C levels in the TLGS population.

\section{Acknowledgements}

This study was supported by the grant SBUMS/M-1391/278 from Shahid Beheshti University of Medical Sciences. The participation of the staff of the Research Institute for Endocrine Sciences and the TLGS unit is gratefully acknowledged. The authors would also like to thank Ms. Niloofar Shiva for the critical editing of the English grammar and syntax of the manuscript.

\section{References}

1. Malaguarnera M, Vacante M, Russo C, et al: Lipoprotein(a) in cardiovascular diseases. Biomed Res Int 2013: 650989, 2013.

2. Azizi F, Rahmani M, Emami H, et al: Cardiovascular risk factors in an Iranian urban population: Tehran lipid and glucose study (phase 1). Soz Praventivmed 47: 408-426, 2002.

3. Gordon T, Castelli WP, Hjortland MC, Kannel WB and Dawber TR: High density lipoprotein as a protective factor against coronary heart disease. The Framingham Study. Am J Med 62: 707-714, 1977.

4. Barter P, Gotto AM, LaRosa JC, et al; Treating to New Targets Investigators: HDL cholesterol, very low levels of LDL cholesterol, and cardiovascular events. N Engl J Med 357: 1301-1310, 2007.

5. Wang $X$ and Paigen B: Genetics of variation in HDL cholesterol in humans and mice. Circ Res 96: 27-42, 2005.

6. Boes E, Coassin S, Kollerits B, Heid IM and Kronenberg F: Genetic-epidemiological evidence on genes associated with HDL cholesterol levels: a systematic in-depth review. Exp Gerontol 44: 136-160, 2009.

7. Holleboom AG, Vergeer M, Hovingh GK, Kastelein JJ and Kuivenhoven JA: The value of HDL genetics. Curr Opin Lipidol 19: 385-394, 2008.

8. Weissglas-Volkov D and Pajukanta P: Genetic causes of high and low serum HDL-cholesterol. J Lipid Res 51: 2032-2057, 2010.

9. Glomset JA: The mechanism of the plasma cholesterol esterification reaction: plasma fatty acid transferase. Biochim Biophys Acta 65: 128-135, 1962 .

10. Jonas A: Lecithin cholesterol acyltransferase. Biochim Biophys Acta 1529: 245-256, 2000. 
11. Safran M, Dalah I, Alexander J, et al: GeneCards Version 3: the human gene integrator. Database (Oxford) 2010: baq020, 2010.

12. McLean J, Wion K, Drayna D, Fielding C and Lawn R: Human lecithin-cholesterol acyltransferase gene: complete gene sequence and sites of expression. Nucleic Acids Res 14: 9397-9406, 1986.

13. Calabresi L, Simonelli S, Gomaraschi M and Franceschini G: Genetic lecithin: cholesterol acyltransferase deficiency and cardiovascular disease. Atherosclerosis 222: 299-306, 2012.

14. Teslovich TM, Musunuru K, Smith AV, et al: Biological, clinical and population relevance of 95 loci for blood lipids. Nature 466 707-713, 2010

15. Haase CL, Tybjærg-Hansen A, Qayyum AA, Schou J, Nordestgaard BG and Frikke-Schmidt R: LCAT, HDL cholesterol and ischemic cardiovascular disease: a Mendelian randomization study of HDL cholesterol in 54,500 individuals. J Clin Endocrinol Metab 97: E248-E256, 2012.

16. Azizi F, Ghanbarian A, Momenan AA, et al: Prevention of non-communicable disease in a population in nutrition transition: Tehran Lipid and Glucose Study phase II. Trials 10: 5, 2009.

17. Azizi F, Raiszadeh F, Salehi P, et al; Tehran Lipid and Glucose Study Group: Determinants of serum HDL-C level in a Tehran urban population: the Tehran Lipid and Glucose Study. Nutr Metab Cardiovasc Dis 12: 80-89, 2002

18. Warnick GR, Benderson J and Albers JJ: Dextran sulfate-Mg2 precipitation procedure for quantitation of high-density-lipoprotein cholesterol. Clin Chem 28: 1379-1388, 1982.

19. Friedewald WT, Levy RI and Fredrickson DS: Estimation of the concentration of low-density lipoprotein cholesterol in plasma, without use of the preparative ultracentrifuge. Clin Chem 18 499-502, 1972.

20. Truett GE, Heeger P, Mynatt RL, Truett AA, Walker JA and Warman ML: Preparation of PCR-quality mouse genomic DNA with hot sodium hydroxide and tris (HotSHOT). Biotechniques 29: 52, 54, 2000.

21. Meyer LR, Zweig AS, Hinrichs AS, Karolchik D, Kuhn RM, Wong M, Sloan CA, Rosenbloom KR, Roe G, Rhead B, Raney BJ, Pohl A, Malladi VS, Li CH, Lee BT, Learned K, Kirkup V, Hsu F, Heitner S, Harte RA, Haeussler M, Guruvadoo L, Goldman M, Giardine BM, Fujita PA, Dreszer TR, Diekhans M, Cline MS Clawson H, Barber GP, Haussler D and Kent WJ: The UCSC Genome Browser database: extensions and updates 2013. Nucleic Acids Res 41: D64-D69, 2013.

22. Rozen S and Skaletsky H: Primer3 on the WWW for general users and for biologist programmers. Methods Mol Biol 132: $365-386,2000$

23. Ye J, Coulouris G, Zaretskaya I, Cutcutache I, Rozen S and Madden TL: Primer-BLAST: a tool to design target-specific primers for polymerase chain reaction. BMC Bioinformatics 13 : 134, 2012.

24. National Institues of Health (NIH): Database of Single Nucleotide Polymorphisms (dbSNP). NIH, Bethseda, MD. http://www.ncbi. nlm.nih.gov/snp. Accessed May, 2013.

25. Flicek P, Ahmed I, Amode MR, et al: Ensembl 2013. Nucleic Acids Res 41: D48-D55, 2013.
26. Altschul SF, Gish W, Miller W, Myers EW and Lipman DJ: Basic local alignment search tool. J Mol Biol 215: 403-410, 1990.

27. Liu K and Muse SV: PowerMarker: an integrated analysis environment for genetic marker analysis. Bioinformatics 21: 2128-2129, 2005

28. Holleboom AG, Kuivenhoven JA, Peelman F, et al: High prevalence of mutations in LCAT in patients with low HDL cholesterol levels in The Netherlands: identification and characterization of eight novel mutations. Hum Mutat 32: 1290-1298, 2011.

29. Stenson PD, Ball E, Howells K, Phillips A, Mort M and Cooper DN: Human Gene Mutation Database: towards a comprehensive central mutation database. J Med Genet 45: 124-126, 2008.

30. Forbes SA, Bindal N, Bamford S, et al: COSMIC: mining complete cancer genomes in the Catalogue of Somatic Mutations in Cancer. Nucleic Acids Res 39: D945-D950, 2011.

31. Sim NL, Kumar P, Hu J, Henikoff S, Schneider G and Ng PC: SIFT web server: predicting effects of amino acid substitutions on proteins. Nucleic Acids Res 40: W452-W457, 2012.

32. Adzhubei IA, Schmidt S, Peshkin L, et al: A method and server for predicting damaging missense mutations. Nat Methods 7: 248-249, 2010.

33. Willer CJ, Sanna S, Jackson AU, et al: Newly identified loci that influence lipid concentrations and risk of coronary artery disease. Nat Genet 40: 161-169, 2008.

34. Aulchenko YS, Ripatti S, Lindqvist I, et al; ENGAGE consortium: Loci influencing lipid levels and coronary heart disease risk in 16 European population cohorts. Nat Genet 41: 47-55, 2009.

35. Kathiresan S, Willer CJ, Peloso GM, et al: Common variants at 30 loci contribute to polygenic dyslipidemia. Nat Genet 41: 56-65, 2009.

36. Khor CC and Goh DL: Strategies for identifying the genetic basis of dyslipidemia: genome-wide association studies vs. the resequencing of extremes. Curr Opin Lipidol 21: 123-127, 2010.

37. Cohen JC, Kiss RS, Pertsemlidis A, Marcel YL, McPherson R and Hobbs HH: Multiple rare alleles contribute to low plasma levels of HDL cholesterol. Science 305: 869-872, 2004.

38. Kathiresan S, Melander O, Guiducci C, et al: Six new loci associated with blood low-density lipoprotein cholesterol, high-density lipoprotein cholesterol or triglycerides in humans. Nat Genet 40: 189-197, 2008.

39. Iyengar SK and Elston RC: The genetic basis of complex traits: rare variants or 'common gene, common disease'? Methods Mol Biol 376: 71-84, 2007.

40. Moriyama K, Sasaki J, Arakawa F, et al: Two novel point mutations in the lecithin:cholesterol acyltransferase (LCAT) gene resulting in LCAT deficiency: LCAT (G873 deletion) and LCAT (Gly344 $\rightarrow$ Ser). J Lipid Res 36: 2329-2343, 1995.

41. Tietjen I, Hovingh GK, Singaraja R, et al: Increased risk of coronary artery disease in Caucasians with extremely low HDL cholesterol due to mutations in ABCA1, APOA1, and LCAT. Biochim Biophys Acta 1821: 416-424, 2012. 